\title{
Exhaled breath condensate magnesium levels of infants with bronchiolitis
}

\author{
Fatma Gül Demirkan¹, Erkan Yılmaz ${ }^{2}$, Melih Hangül1 ${ }^{1}$, Didem Öztürk ${ }^{1}$, Hasan Demirkan ${ }^{1}$, \\ Mustafa Soylak², Mehmet Köse ${ }^{1}$ \\ ${ }^{1}$ Division of Pediatric Pulmonology, Department of Pediatrics, Faculty of Medicine and ${ }^{2}$ Department of Chemistry, Faculty \\ of Sciences, Erciyes University, Kayseri, Turkey. \\ E-mail: mhmtkose@yahoo.com \\ Received: 6th December 2017, Revised: 16th January 2018, Accepted: 18th January 2018
}

SUMMARY: Demirkan FG, Yılmaz E, Hangül M, Öztürk D, Demirkan H, Soylak M, Köse M. Exhaled breath condensate magnesium levels of infants with bronchiolitis. Turk J Pediatr 2018; 60: 535-539.

The aim of this study is to determine the exhaled breath condensate (EBC) magnesium levels of infants with bronchiolitis and to investigate their relationship with disease severity. Fifty infants with moderate and severe bronchiolitis, grouped according to the bronchiolitis clinical severity scores (CSS), and 25 healthy children were included in the study. EBC was collected using an $\mathrm{R}$ tube commercial device; flammable atomic absorption spectrometry was used for the identification of magnesium level in this method. The median concentration of EBC magnesium levels in the group of moderate bronchiolitis patients was $0.82(0-2.71) \mu \mathrm{g} / \mathrm{ml}$, and that of severe bronchiolitis patients was $0.57(0-3.16) \mu \mathrm{g} / \mathrm{ml}$. The median concentration of EBC magnesium levels in the control group was $0.45(0.06-2.66) \mu \mathrm{g} / \mathrm{ml}$. There were no significant differences among the three groups $(\mathrm{p}>0.559)$. The magnesium levels of the two bronchiolitis groups were also found in a wide range. In conclusion, EBC magnesium levels were not different in bronchiolitis patients and do not reflect disease severity.

Key words: bronchiolitis, exhaled breath condensate, magnesium.

Bronchiolitis is the most common acute viral infection of the respiratory system in the world, involving the terminal and respiratory bronchioles, and is usually seen in children under the age of two. The pathophysiological changes resulting in bronchiolitis are acute inflammation, edema, necrosis of small airway epithelial cells, increased mucus production and bronchospasm. ${ }^{1,2}$ Bronchiolitis is a clinical diagnosis which is made without help from diagnostic tests. Acute bronchiolitis is a selflimiting viral disease and usually does not require aggressive treatments. Treatment is mainly based on supportive care. ${ }^{3}$ Patients with prematurity, bronchopulmonary dysplasia, congenital heart disease and immunodeficiency have a higher incidence of acute bronchiolitis and are more severely affected than healthy children ${ }^{4}$. However, in some infants with bronchiolitis who have no risk factor, the disease is more severe and its cause is unknown.

Magnesium is an important intracellular cation. It functions as a cofactor in the activation of many enzyme systems.5,6 In addition, magnesium inhibits the contraction of bronchial smooth muscle cells and plays an important role in regulating bronchial reactivity. It acts as a calcium antagonist in membrane channels and intracellular regions. In addition, it suppresses calcium activation in the sarcoplasmic reticulum and limits calcium excretion from the sarcoplasmic reticulum, which is the main calcium store in the cell. ${ }^{7}$ It also bronchodilates smooth muscle cells by increasing histamine and acetylcholine release. With these mechanisms, magnesium regulates the level of intracellular calcium and affects 
smooth muscle tonus. ${ }^{8}$ It was shown that intravenous and inhaled magnesium sulfate therapy can be used safely to improve the clinical symptoms of bronchoconstriction and acute asthma ${ }^{9,10}$. Previously, we demonstrated that nebulized magnesium sulfate plus salbutamol treatment were effective for improving the clinical scores of patients with bronchiolitis. ${ }^{11}$ Our findings suggested that there may be a relationship between magnesium deficiency and bronchiolitis because of the good response of bronchiolitis patients to magnesium treatment.

Exhaled breath condensate (EBC) aerosols and vapors are collected noninvasively. They can reflect the physiological and pathological processes in the lung. ${ }^{12}$ The severity of certain pulmonary diseases and the response to treatment may provide information on the measurement of several markers in the EBC. ${ }^{13}$ The exhaled aerosol droplets are assumed to originate from the extracellular surface fluid layer and may reflect the composition of the bronchoalveolar surface fluid. This may help to provide us with information about the tissue magnesium levels of patients.

The aim of this study is first of all to determine whether there is a difference in the EBC magnesium levels of infants with bronchiolitis and control groups. Secondly, we tried to determine the relationship of $\mathrm{EBC}$ magnesium levels with bronchiolitis clinical severity scores.

\section{Material and Methods}

This prospective study was conducted between January 2016 and May 2016 at the Division of Pediatric Pulmonology Erciyes University Hospital with 75 infants whose ages were between 2-24 months. Fifty of these infants who were admitted to the Division of Pediatric Pulmonology with complaint of wheezing and diagnosed with bronchiolitis were eligible for the study. The other 25 infants, who were completely healthy and applied to the outpatient pediatric clinic, were eligible for the control group. They were separated into three groups. Group 1: children with moderate bronchiolitis $(n=25)$; Group 2: children with severe bronchiolitis $(n=25)$; and Group 3: clinically healthy infants as a control group $(\mathrm{n}=25)$.

Bronchiolitis was diagnosed by clinical history, respiratory symptoms, physical examination and/or chest X-ray findings. Bronchiolitis clinical severity scoring (CSS) was performed in diagnosed cases. The CSS was defined based on four parameters including respiratory rate, degree of wheezing, degree of accessory muscle use, and general condition, as described previously by Wang et al. ${ }^{14} \mathrm{~A}$ single point was given to patients with a respiratory rate of 31-45 breaths/min, wheezing at terminal expiration using a stethoscope, intercostal retraction and normal general condition. Two points were given to patients with a respiratory rate of 46-60 breaths/min, wheezing during the entire expiration or audible on expiration without stethoscope, tracheosternal retractions and stable general conditions. Three points were given to patients with a respiratory rate of $>60$ breaths/min, inspiratory and expiratory wheezing without stethoscope and severe retraction with nasal flaring and a general condition of irritability, lethargy and poor appetite. CSS 1-3 was considered as mild bronchiolitis, CSS 4-8 as moderate bronchiolitis and CSS 9-12 as severe bronchiolitis. ${ }^{14}$

Exclusion criteria were as follows: infants with CSS $<4$, cardiopulmonary or neurological disease, prematurity, history of recurrent wheezing episodes, history of atopy, proven immune deficiency, age $<2$ months or $>2$ years, and previous treatment with bronchodilators or corticosteroids. Signed informed consent was obtained from the parents of each infant and the study was approved by the Ethics Committee of Erciyes University. Clinical information and findings of the physical examinations of patients were collected by creating a standard form. The age, sex, physical examination findings and bronchiolitis clinical severity score of each patient were recorded.

EBC collection is a noninvasive process that is both simple and safe. When the exhalation air meets a cooled surface and falls below the dew point, the aerosolized liquid particles condense on top of the $\mathrm{R}$ tube wall. ${ }^{12} \mathrm{An}$ $\mathrm{R}$ tube device (Portable EBC Collection Devices Respiratory Research Inc, Charlottesville, USA) was used to collect the EBC and the procedure was conducted in accordance with the EBC collection rules published by the American Thoracic Society/ European Respiratory Society (ATS/ERS) ${ }^{15}$ A mask suitable for children was selected and the children breathed in tidal 
volume for 10 minutes through the mouth of the R-tube. In this way 0.5-1.0 ml exhaled air was collected in sterile tubes and magnesium levels were measured on the same day.

EBC samples were studied at Erciyes University Science Faculty's Chemistry Laboratory. Flammable atomic absorption spectrometry, by means of a Perkin Elmer device (an Analyst 300 model), was used for the identification of magnesium level in the method. The calibration curve generation method was used to determine the magnesium concentration in the samples. In this method, magnesium standard solutions were prepared at increasing concentration and the magnesium concentration in the samples was calculated using a calibration line.

Statistical analysis was performed using SPSS (Statistical Package for Science Studies) version 22.0 for Windows. The normality of data distribution was assessed by the ShapiroWilk test and Q-Q histogram graphics. Since the magnesium levels were not normally distributed, comparisons between groups were made using the non-parametric Mann-Whitney $\mathrm{U}$ and Kruskal-Wallis tests. A p value $<0.05$ was considered as significant.

All procedures were approved by the Ethics Committee of Erciyes University (96681246/195). This study was funded by the Scientific Research Projects Unit of Erciyes (Project code: TTU-2016-6661).

\section{Results}

Twenty five patients with moderate bronchiolitis and 25 patients with severe bronchiolitis were enrolled in this study. For the control group, 25 healthy children were included. No significant differences were detected among the three groups for age and sex $(p=0.216$, $\mathrm{p}=0.146$; Table I). The CSS median value of patients with moderate bronchiolitis was $5.8 \pm 1$. and that of severe bronchiolitis patients was $10.0 \pm 0.9(\mathrm{p}<0.001 ;$ Table I).

Magnesium concentration was measured in all samples (Table I). There was no significant difference among the three groups for EBC magnesium levels $(p=0.559)$. The median concentrations of EBC magnesium level of the moderate and severe bronchiolitis groups were comparable to that of the control group $(p=0.554, p=0.219$ respectively, Table I). The median concentrations of EBC magnesium level of the moderate and severe bronchiolitis patient groups were comparable, as well $(\mathrm{p}=0.463$, Table I).-

\section{Discussion}

Our preliminary study showed that magnesium level could be measured in the EBC of patients with bronchiolitis. Our study results indicate that EBC magnesium levels had a broad distribution in infants with bronchiolitis. In our clinical observations, some patients do not respond to magnesium treatment while others do. The reason for this is not fully known at present. There might be an association between magnesium levels and magnesium therapy. Further studies on this subject are needed. No significant differences were observed between the EBC magnesium levels in infants with bronchiolitis (moderate and severe) and control groups. To the best of our knowledge, this is the first study in the literature to evaluate the EBC magnesium levels of infants with bronchiolitis.

In some infants with bronchiolitis who have no risk factor, the disease is more severe. However, we do not know exactly why. Magnesium, in addition to its bronchodilator effect, also plays a role in inflammation, oxidative stress and the apoptosis process. Decreased magnesium intake results in an increase in cytokines and reactive oxygen species, increased phagocytosis and the start of the pro-inflammatory process ${ }^{16}$. Magnesium deficiency may be related with increased inflammatory process and smooth muscle contractility. This may be lead to the disease being more severe. Recent findings show that inhaled magnesium sulfate combined with salbutamol therapy is significantly effective for the treatment of acute bronchiolitis and improvement in clinical scores. In our previous study, combination therapy with inhaled magnesium sulfate and salbutamol was significantly $(p<0.05)$ more effective than treatment with inhaled magnesium sulfate or salbutamol alone for patients with acute bronchiolitis attack. ${ }^{11}$ In recent years, the magnesium metabolism in asthma and bronchiolitis has been studied. Generally, in these studies magnesium sulfate was used for treatment. ${ }^{11,17}$. There was no study about the magnesium levels of infant bronchiolitis when 
Table I. Baseline Characteristics, Clinical Severity Scores and Exhaled Breath Condensate Magnesium Levels According to Study Groups.

\begin{tabular}{lcccc}
\hline \multirow{2}{*}{ Findings } & \multicolumn{3}{c}{ Groups } & \\
\cline { 2 - 4 } & $\begin{array}{c}\text { Moderate bronchiolitis } \\
(\mathrm{n}=25)\end{array}$ & $\begin{array}{c}\text { Severe bronchiolitis } \\
(\mathrm{n}=25)\end{array}$ & $\begin{array}{c}\text { Controls } \\
(\mathrm{n}=25)\end{array}$ & P value \\
\hline Age, months & $7.8 \pm 4.1$ & $7 \pm 5.4$ & $8.56 \pm 4.95$ & 0.216 \\
Male/female & $17 / 8$ & $14 / 11$ & $10 / 15$ & 0.146 \\
EBC magnesium, $\mu \mathrm{g} / \mathrm{ml}$ & $0.82(0-2.71) *_{\mathrm{x}}$ & $0.57(0-3.16)+\mathrm{x}$ & $0.45(0.06-2.66)$ & 0.559 \\
CSS & $5.8 \pm 1.0$ & $10.0 \pm 0.9$ & & $<0.001$ \\
\hline
\end{tabular}

Data is presented as mean \pm standard deviation or median (minimum-maximum) as appropriate

CSS: Clinical Severity Scores, EBC: exhaled breath condensate

${ }^{*} \mathrm{p}=0.554$ comparison between moderate bronchiolitis and control group

$+p=0.219$ comparison between severe bronchiolitis and control group

${ }^{\mathrm{x}} \mathrm{p}=0.463$ comparison between moderate and severe bronchiolitis groups

we reviewed the literature. However, there were a few studies on asthma. The magnesium level is generally evaluated by measuring serum levels of magnesium and there are a few studies at tissue level ${ }^{16,18}$. Because serum magnesium measurement does not reflect the magnesium levels in the body, researchers tend to measure levels of magnesium in cells such as leukocytes, and erythrocytes. Studies evaluating the level of magnesium in the serum and intracellular levels (red blood cell, lymphocytes etc.) of asthma are available in the literature. Hashimoto et al. ${ }^{19}$ reported in their study that EBC magnesium level is inversely proportional to nonspecific bronchial reactivity. In another study there was no significant difference between the plasma and EBC magnesium levels of patients with chronic obstructive pulmonary disease and the control group. ${ }^{20}$ In the study of Dodig et al, ${ }^{16}$ the authors reported that no difference was recorded for either EBC magnesium or calcium concentration between the asthma and control groups. However, the magnesium ratio to calcium in asthma was also found to be statistically significantly lower compared to the control group. In our study, only magnesium levels were assessed in the EBC of patients with acute bronchiolitis, which can be considered as one of the limitations of our study. We speculate that the magnesium/calcium ratio might be a predictor for detecting the severity of bronchiolitis.

The concentration of magnesium in the body can not be accurately assessed by the determination of serum magnesium concentration. Thus, in this study, we evaluated EBC magnesium levels instead of plasma levels which may reflect lung magnesium composition better than plasma levels. Our study was the first to investigate EBC concentrations of magnesium in living tissue for this age group.

This study has several limitations. First the study sample size is small. Secondly; although, collection of EBC is a non-invasive and simple method of sampling the respiratory tract, there are several factors that influenced the EBC composition such as breathing pattern, exhaled breath volume per minute, mouth versus nose breathing, ambient conditions, dilution, etc. ${ }^{15}$ The breathing pattern and breath volume per minute of each infant with bronchiolitis is not the same and variations in these may significantly influence EBC composition. The difference in respiratory pattern, and breath volume per minute for each child studied resulted in relatively small volumes of respiratory droplets. This may cause diagnostic difficulty for the measurement of magnesium from EBC.

In conclusion, the magnesium level in EBC of infants and young children with bronchiolitis was measured by a non-invasive method but no relationship was found between the magnesium level in the EBC and severity of bronchiolitis. Further studies are needed to provide more data on this subject.

\section{REFERENCES}

1. Ali S, Plint AC, Klassen TP. Bronchiolitis. In: Wilmott RW, Kendig EL, Boat TF, Bush A, Chernick V (eds). Kendig and Chernick's Disorders of the Respiratory Tract in Children (8th ed.). Philadelphia: Elseiver Saunders, 2012: 443-452. 
2. Midulla F, Nicolai A, Moretti C. Acute viral bronchiolitis. In: Eber E, Midulla F (eds). ERS Handbook of Paediatric Respiratory Medicine (1st ed). United Kingdom: European Respiratory Society, 2013: 305-309.

3. Lieberthal AS, Bauchner CH, Hall CB, et al; Subcommittee on Diagnosis and Management of Bronchiolitis. Diagnosis and management of bronchiolitis. Pediatrics 2006; 118: 1774-1793.

4. Shay DK, Holman RC, Roosevelt GE, Clarke MJ, Anderson LJ. Bronchiolitis-associated mortality and estimates of respiratory syncytial virus-associated deaths among US children, 1979-1997. J Infect Dis 2001; 183: 16-22.

5. Dominguez LJ, Barbagallo $\mathrm{M}$, Di Lorenzo $\mathrm{G}$, et al. Bronchial reactivity and intracellular magnesium: A possible mechanism for the bronchodilating effects of magnesium in asthma. Clin Sci (Lond) 1998: 95; 137-142.

6. Mathew R, Altura BM. Magnesium and the lungs. Magnesium 1988; 7: 173-187.

7. Gourgoulianis KI, Chatziparasidis G, Chatziefthimiou A, Molyvdas PA. Magnesium as a relaxing factor of airway smooth muscles. J Aerosol Med 2001; 14: 301307.

8. Rolla G, Bucca C, Caria E. Dose-related effect of inhaled magnesium sulfate on histamine bronchial challenge in asthmatics. Drugs Exp Clin Res 1988; 14: 609-612.

9. Su Z, Li R, Gai Z. Intravenous and nebulized magnesium sulfate for treating acute asthma in children: A systematic review and meta-analysis. Pediatr Emerg Care 2018; 34: 390-395

10. Powell C, Dwan K, Milan SJ, et al. Inhaled magnesium sulfate in the treatment of acute asthma. Cochrane Database Syst Rev 2012; 12: CD003898.

11. Kose M, Ozturk MA, Poyrazoğlu H, et al. The efficacy of nebulized salbutamol, magnesium sulfate, and salbutamol/magnesium sulfate combination in moderate bronchiolitis. Eur J Pediatr 2014; 173: 1157 1160.
12. Hunt J. Exhaled breath condensate: An evolving too for noninvasive evaluation of lung disease. J Allergy Clin Immunol 2002; 110: 28-34.

13. Kazani S, Planaguma A, Ono E, et al. Exhaled breath condensate eicosanoid levels associate with asthma and its severity. J Allergy Clin Immunol 2013; 132: 547-553.

14. Wang EE, Milner RA, Navas L, Maj H. Observer agreement for respiratory signs and oximetry in infants hospitalized with lower respiratory infections. Am Rev Respir Dis 1992; 145: 106-109.

15. Horvath I, Hunt J, Barnes PJ, et al. Exhaled breath condensate: Methodological recommendations and unresolved questions. Eur Respir J 2005; 26: 523-548.

16. Dodig S, Vlasić Z, Cepelak I, Topić RZ, Turkalj $\mathrm{M}$, Nogalo B. Magnesium and calcium in exhaled breath condensate of children with asthma and gastroesophageal reflux disease. J Clin Lab Anal 2009; 23: 34-39.

17. Hughes R, Goldkorn A, Masoli M, Weatherall M, Burgess C, Beasley R. Use of isotonic nebulised magnesium sulphate as an adjuvant to salbutamol in treatment of severe asthma in adults: Randomized placebo-controlled trial. Lancet 2003; 361: 2114-2117.

18. Banović S, Navratil M, Vlašić Z, Topić RZ, Dodig S Calcium and magnesium in exhaled breath condensate of children with endogenous and exogenous airway acidification. J Asthma 2011; 48: 667-673.

19. Hashimoto Y, Nishimura Y, Maeda H, Yokoyama M Assessment of magnesium status in patients with bronchial asthma. J Asthma 2000; 37: 489-496.

20. Effros RM, Peterson B, Casaburi R, et al. Epithelial lining fluid solute concentrations in chronic obstructive lung disease patients and normal subjects. J Appl Physiol (1985) 2005; 99: 1286-1292. 